\title{
URC97166
}

\section{Dexterity-enhanced Telerobotic Microsurgery}

\author{
Steve Charles \\ MicroDexterity Systems, Inc. and Charles Retina Institute, Memphis, TN \\ Hari Das, Timothy ohm, Curtis Boswell, Guillermo Rodriguez, Robert Steele \\ J et Propulsion Laboratory, California Institute of Technology, Pasadena, CA. \\ Dan Istrate \\ California Institute of Technology, Pasadena, CA.
}

\section{Introduction}

The work reported in this paper is the result of a collaboration between researchers at the J et Propulsion Laboratory and Steve Charles, MD, a vitreo-retinal surgeon. The Robot Assisted MicroSurgery (RAMS) telerobotic workstation developed at JPL [9] is a prototype of a system that will be completely under the manual control of a surgeon. The systcm, shown on Figure 1, has a slave robot that will hold surgical instruments. The slave robot motions replicate in six degrees of freedom those of the surgeon's hand measured

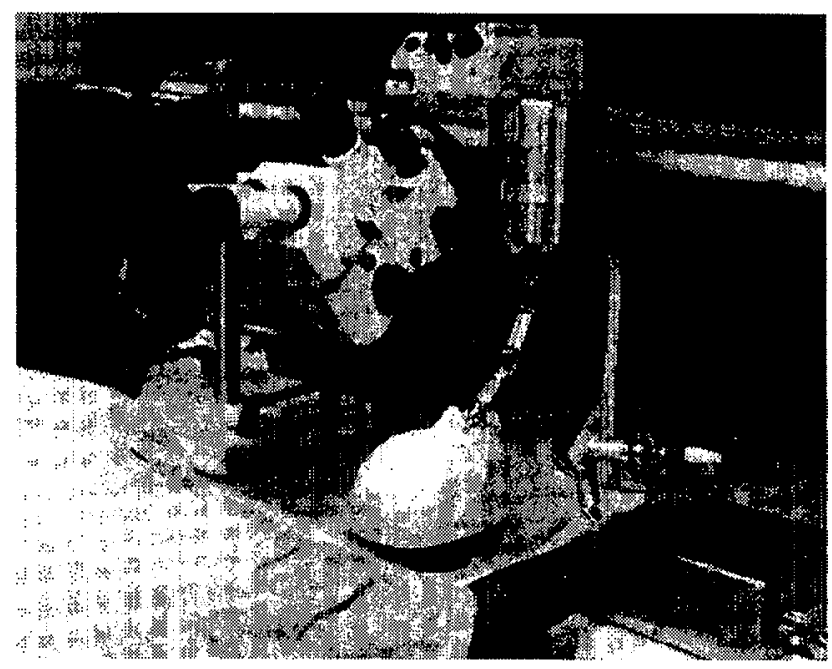

Figure 1: RAMS telerobot system.

using a master input device with a surgical instrument, shaped handle. The surgeon commands motions for the instrument by moving the handle in the desired trajectories. The trajectories are measured, filtered, and scaled down then used to drive the slave robot,.

We present the details of this telerobotic system by first giving an overview of the subsystems and their interactions in the following section then present det ails in subsequent subsections divided according to subsystem. This paper concludes wit $\mathrm{h}$ a description of a recent demonstration of a simulat cd microsurgery procedure performed at JPL. 


\section{System description}

Figure 2 shows an overview of' the hardware components of the RAMS telerobotic system.

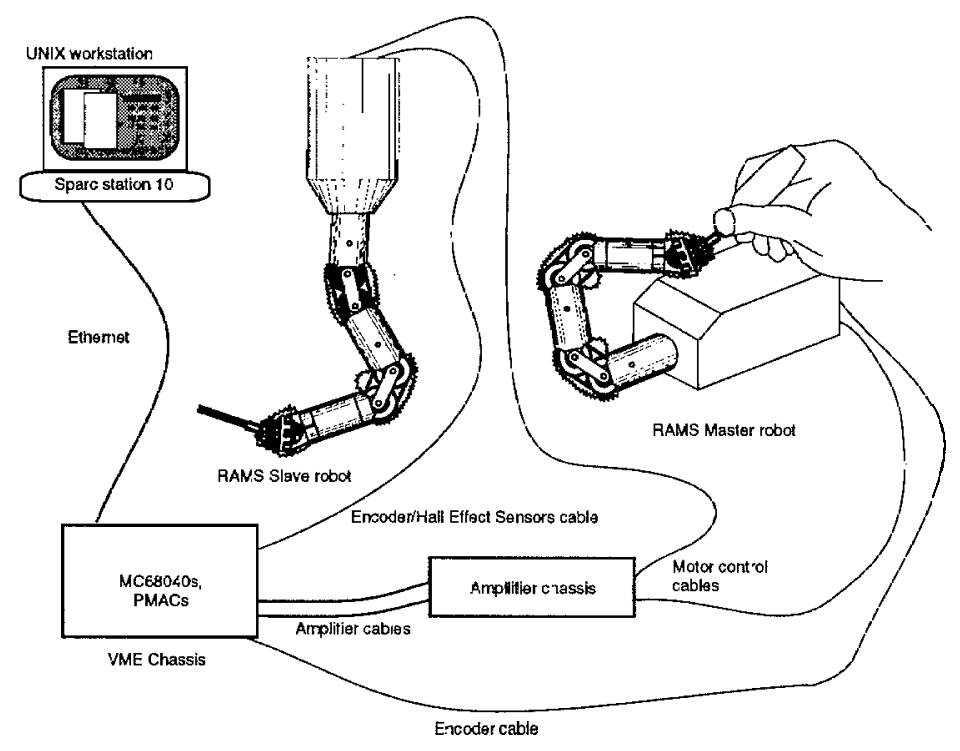

Figure 2: RAMS slave robot system.

Components of the RAMS system have been categorized into four subsystems. They arc the mechanical subs ystem, the electronics subsystem, the servo-control subsystem and the high-level software subs yst em. The mechanical subsystem consists of a master input device and a slave robot arm with associated motors, encoders, gears, cables, pulleys and linkages that cause the tip of the robot to move under computer control and to measure the surgeon's hand motions precisely. The electronics subsystem consists of the motor amplifiers, a safety electronics circuit and relays within the amplifier box shown on Figure 2 . These elements of the subsystcm ensure that a number of error conditions are handled gracefully. The servo-control subsystem is implement ed in hardware and software. The relevant hardware parts of the subsystem are the servo-control boards and the computational processor boards. Servo-control software functions include setting-up the control parameters and running the servo-loop on the servo-cent rol board to control the six motors, implementing the communication between the computation and servo-control boards, initializing the servo-control system and communicating with the electronics subsystem and communicant ing with the high-level software subsystem. The high-level software subsystem interfaces with a user, controls initialization of the system software and hardware, implements a number of demonstration modes of robot cent rol and computes both the forward and inverse kinematics. A drawing of the interaction between the subs yst cms oft he RAMS slave robot, is shown on Figure 3.

\subsection{Mechanical subsystem}

The RAMS slave manipulator is a six degrees-of-freedom tendon-driven robotic arm designed to be compact yet exhibit very precise 10 micron relative positioning capability as well as maintain a very high work volume, Physically, the arm measures $2.5 \mathrm{~cm}$. in diameter and is $25.0 \mathrm{~cm}$. Iong from its base to tip. It is mounted to a cylindrical base housing which measures $12 \mathrm{~cm}$. in diameter by $18 \mathrm{~cm}$ long that contains all of the drives that actuate the arm. A photograph of the arm appears on Figure 4. The joints of the arm are a torso joint rotating about an axis aligned with the base axis and positioned at the point the arm emerges from its base, a shoulder joint rotating about two axes that are in the same plane and perpendicular to the preceding links, an elbow joint that also rotates about two axes that are in the same plane and perpendicular to the preceding links, and a wrist with pitch, yaw and roll joints. 


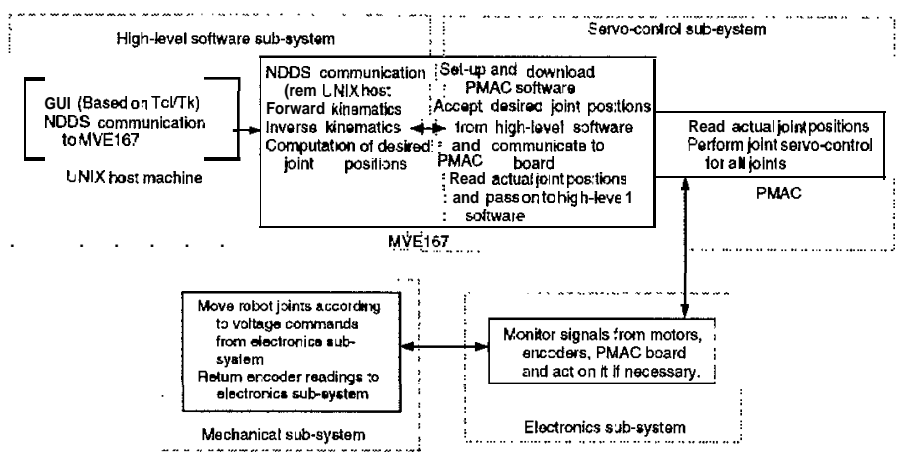

Figure 3: Sub-systems of the RAMS telerobot system.

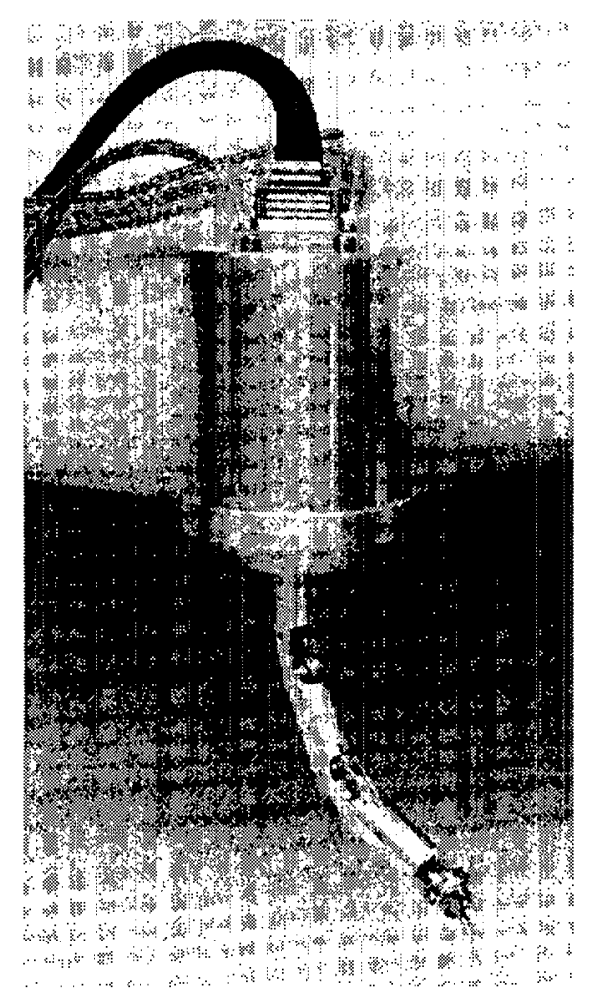

Figure 4: RAMS slave robot.

The master device, cinematically similar to the slave robot, also has six tendon driven joints. It is 2.5 $\mathrm{cm}$. in diameter and $25 \mathrm{~cm}$. long. Its base houses high-resolution optical encoders requiring a larger volume - a box of size $10.8 \mathrm{~cm}$ by $18.4 \mathrm{~cm}$ by $23.5 \mathrm{~cm}$. Gear transformation ratios in the master arm are reduced to allow backdrivability. A photograph of the master input device is shown on Figure 5.

The slave wrist design (based on the kinematics of the Rosheim OMNI-WRIST [8]) utilizes a dual universal joint to give a three degrees-of-freeclom, singularity free, mechanically decoupled joint that operates in a full hemisphere of motion (up to 90 degrees in any direction). The master wrist design uses a universal joint to transmit rotation motion through the joint while allowing pitch and yaw motions about the joint resulting in singularity free motion over a smaller range of motion in three degrees-of-freedom. The fourth and fift $h$ axes of the master and slave robots arc unique joints that rotate about 2 axes and allow passage of cables to pass through the joint for actuating the succeeding joints without affecting their cable lengths. The sixth axes are torso joints which simply rot ates the manipulators relative to their base housing, $\mathrm{F}$ or $\mathrm{t}$ he slave robot 


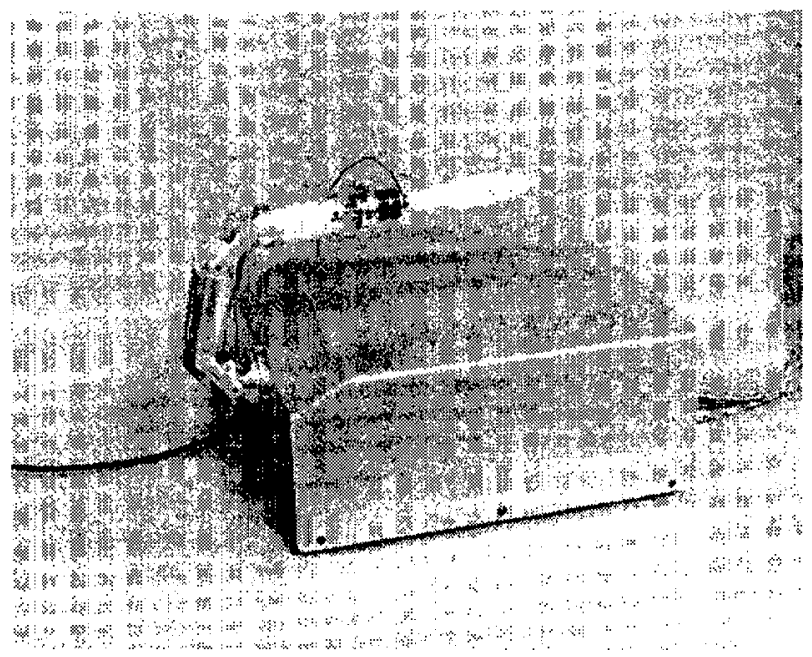

Figure 5: RAMS Master input device.

the torso range of motion is 330 degrees while on the master it is 30 degrees.

\subsection{Electronics subsystem}

The RAMS electronics subsystem design includes off the shelf and custom designed electronics. Figure 6 shows a layout of its general components.

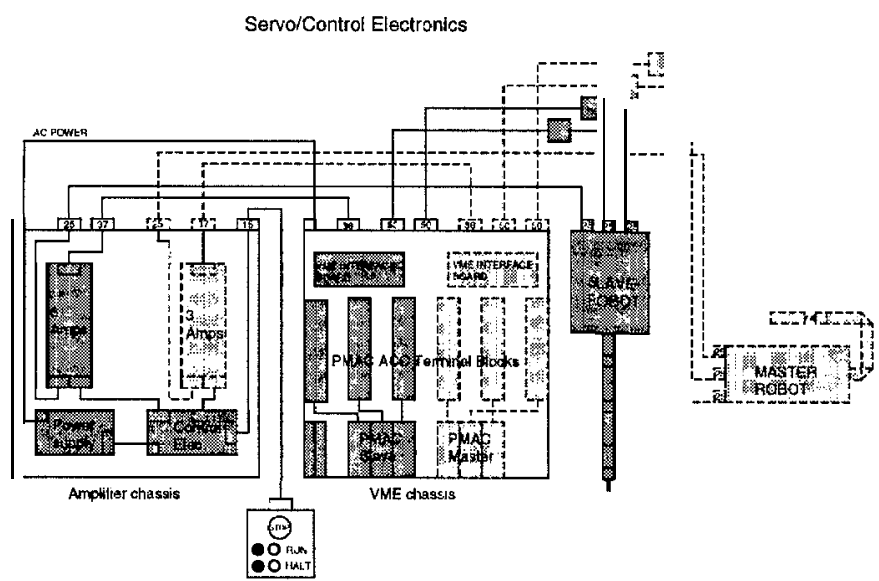

Figure 6: Electronics components and cabling.

Components of the electronics subsystem are a VME chassis, an amplifier chassis and safety electronics. The VME chassis houses the VME backplane and two Motorola MVME-167 computer boards used for high level system control. The VME chassis also contains the PMAC servo control cards and six supporting interface modules, power supplies $(+/-15 \mathrm{v})$ and a cable interface board. The VME chassis front panel contains main power control $(A C)$ for the system. The rear panel provides access to the cent rol computers serial communications port (RS-232).All components above are off the shelf items except the cable interface board. 


\subsection{Servo-control subsystem}

The RAMS servo-control system is implemented on processor boards and servo-control boards installed in a VME chassis. Two Motorola MVME-167 boards, named Proc0 and Proc1, are installed on the VME chassis and run under the VxWorks operating system, Proc0 performs kinematic, communication and high-level control functions. These functions are described in the High Level Software Archit ecturc Section. Calls to subroutines that read and set joint angle positions of the robot arc made from the high-level real-time software on Proc0. These routines, through shared memory implemented between Proc 0 and Proc1, provide setpoints and read current joint angles of the robot. Proc1, in turn, passes the setpoints for controlling the robot to the servo control board and retrieves the joint angles measured by the servo-control board. The servo level control system uses the PMAC-VME board by Delta Tau.

Communication between Proc0,Proc1 and the PMAC-VME boards is through shared memory. The PMAC board has a large variety of features for motor control, with a customer base largely from industrial installations. The key features used for control of the RAMS robot include:

\subsection{High-level software subsystem}

There arc a number of components to the high-level software for the RAMS slave robot. A drawing of the parts of the software in shown on Figure 7. Embedded in the computational blocks of the real-time

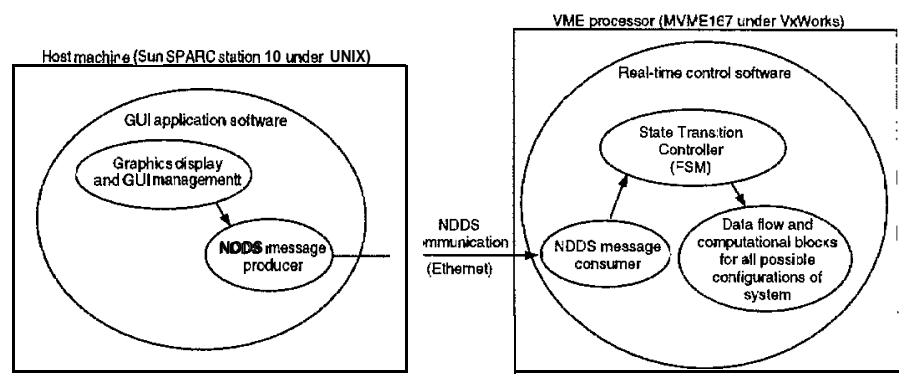

Figure 7: Parts of the high-level software.

control software are the kinematic control algorithms. They are based on algorithms developed at JPL $[6],[7]$ for the unique geometry of the robot. The demonstration of different control modes of the robot was implemented using a software development tool for real-time s ystems called Cent rol Shell [3], [4]. Handling of operator commands in the real-time software, transitions between states of control, changes in data flow due to transitions of states in the software and the algorithms executed within computation blocks. The user specifies the control modes of the system through a graphic user interface (GUI) implemented with Tcl/Tk [2]. Commands entered into the GUI are transmitted over an Ethernet connection and are reccived on the real-time software side of the system. The message passing between the 2 parts of the software system uses NDDS [5]. A producer part creates the messages and broadcasts them from the GUI part of the system and a consumer part receives the messages and processes them.

\section{Simulated Surgery}

In September of 1996, a demonstration of a simulated eye microsurgery procedure was successfully conducted using the RAMS t elerobotic system. The procedure demonstrated was the removal of a microscopic 0.015 inch diameter particle from a simulated eyeball.

Features added to the RAMS system to enable successful performance of the eye surgery demonstration were foot switch operated indexed motion, a surgical instrument mounted on the slave robot tip and a pivoting shared control algorithm to automatically compensate for pitch and yaw orientation of the surgical 
instrument while the operator cent rols the $x-, y-, z$ - and roll motions of $t$ he instrument. Figure 8 shows the RAMS system as seen performing the simulated eye microsurgery procedure.

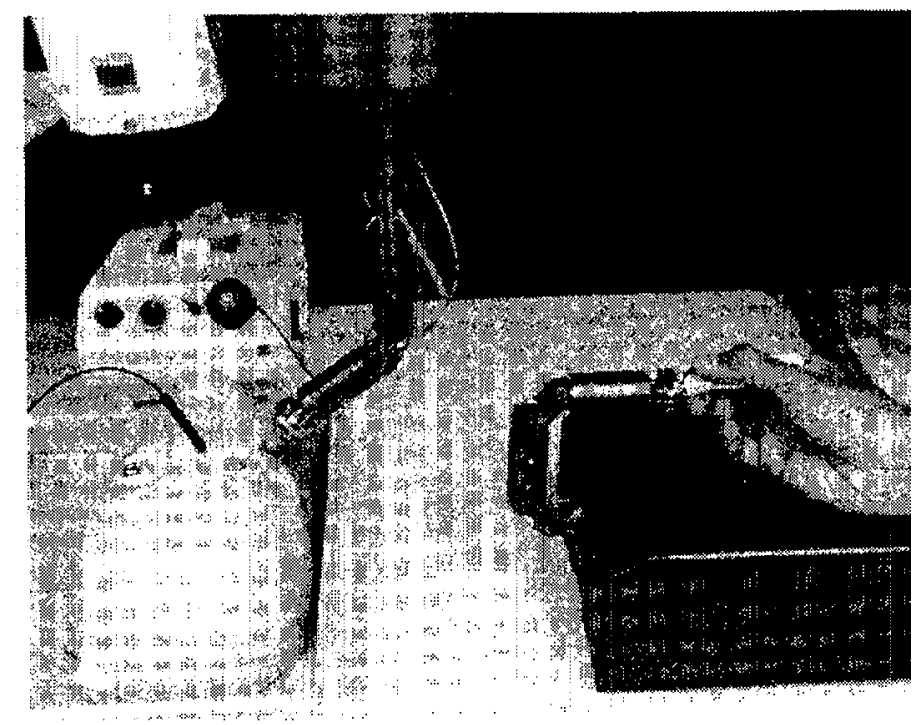

Figure 8: Performing the eye surgery demonstration.

In the next year, the RAMS systcm will be upgraded to implement force feedback to the master arm from force sensors mounted on the slave robot. In addition, experiments will be conducted to characterize the performance of the system as compared to direct manual manipulation in simulations of microsurgical tasks.

\section{References}

[1] Charles, S. "Dexterity Enhancement for Surgery", in Computer Integrated Surgery: Technology and Clinical Applications, ed. R. H. Taylor, S. Lavalle, G. Burdea, R. Mosges, MIT Press, Cambridge, MA 1996.

[2] Ousterhout, J.K. "Tcl and the Tk Toolkit", Addison Wesley, Reading,Mass. 1994.

[3] Real-time Innovations, Inc., "Control Shell Programmer's Reference Manual Vol. 1", Sunnyvale, CA, 1995.

[4] Real-time Innovations, Inc., "Control Shell Programmer's Reference Manual Vol. 2", Sunnyvale, CA, 1995.

[5] Real-time Innovations, Inc., "NDDS Programmer's Reference Manual", Sunnyvale, CA, 1995,

[6] Rodriguez, G., K. Kreutz, and A. Jain, "A Spatial Operator Algebra for Multibody System Dynamics," The J ournal of the Astronautical Sciences, Vol. 40, No. 1, pp. 27-50, J anuary-March 1992.

[7] Rodriguez, G., "Kalman Filtering, Smoothing, and Recursive Robot Arm Forward and Inverse Dynamics," IEEE Transactions on Robotics and Automation, Vol. 3, pp. 624-639, Dec. 1987.

[8] Rosheim, M. E., “Robot Wrist Actuators", J ohn Wiley \& Sons., New York, 1989.

[9] Schenker, P., Das, H., and Ohm, T. ".4 new robot for high dexterity microsurgery" Proceedings of the First International Conference, CVRMed '95, Nice, France April, 1995. also in Computer Vision, Virtual Reality and Robotics in Medicine, Lecture Notes in Computer Science, Ed. Nicholas Ayache, Springer-Verlag, Berlin 1995.

[10] Williams III, R. L., "F orward and Inverse Kinematics of Double Universal Joint Robot Wrists," Space operations Applications and Research (SOAR) Symposium, Albuquerque, NM, J une 26-28, 1990,

\section{Acknowledgment}

This work was carried out at the Jet Propulsion Laboratory under contract with the National Aeronautics and Space Administration. The authors affiliated with JPL are in the Automation and Control Section, J et Propulsion Laboratory, California Institute of Technology, 4800 Oak Grove Drive, Pasadena, CA 91109. Steve Charles, MD is the CEO of MicroDexterity Systems, Inc. 